\title{
The influence of the riser dimensions on the effectiveness of feeding solidifying cast elements
}

\author{
Leszek Sowa ${ }^{1, *}$, Tomasz Skrzypczak ${ }^{1}$, Pawet Kwiatoń ${ }^{1}$ \\ ${ }^{1}$ Czestochowa University of Technology, Institute of Mechanics and Machine Design Fundamentals, \\ Dąbrowskiego 73, 42-201 Częstochowa, Poland
}

\begin{abstract}
The mathematical model and numerical simulation results of metals alloy solidification process based on the finite element method (FEM) are presented in this paper. After completion of the mould filling process the main solidified of molten metal takes place and its shrinkage. The phenomenon of casting shrinkage cannot be avoided. However, it is possible to minimize the occurrence of its negative effects on the casting quality. The phenomenon of shrinkage defects formation was included in the numerical calculations and they were tried to reduce them. It is important aspect of this work which makes possible the prediction of the location of the casting defect depending on shape of the riser. The results of computer calculations of the solidification process of the three-dimensional casting together with the conical or cylindrical riser are discussed in detail and presented.
\end{abstract}

Keywords: numerical modelling, solidification, casting defect, FEM

\section{Introduction}

Constantly increasing customer demands for the production of the high-quality castings result in the intensive technological development of their production methods demanding continuous researches. These studies are mainly aimed at obtaining castings without the casting defects of the high strength properties or creating the layered castings that can work in high temperature conditions or be resistant to corrosion [1-3]. Research on real objects is difficult due to the high temperatures occurring there, which is why computer simulations become the basic way of technological improvement of foundry methods [4-10]. For the analysis of the casting process must be formulated appropriate mathematical models that would take into account as many phenomena as possible occurring in the process of casting formation. Considerations are usually limited to selecting the most important phenomena for the process due to the difficulty in obtaining an effective numerical solution. In mathematical models, the influence of molten metal movements on the solidification process is usually

\footnotetext{
* Corresponding author: sowa@imipkm.pcz.pl

Reviewers: Marek Macko, Milan Sága
} 
included [6-9] or neglected $[4,5,10]$, but then a mechanical phenomenon (gap formation) and shrinkage phenomenon causing the casting defects are considered $[2-5,7-9,11,12]$.

The number of defects formed in the casting is closely related to the parameters of the molten metal pouring and the design of gating system. Another reason of the formation of these defects is the shrinkage of the metal. As the solidification process progresses, the particular parts of the casting should be evenly fed from the riser by liquid metal. In the case when the feeding by liquid metal of the solidifying part of the casting is interrupted the empty spaces or macro-porosities are created which reduce the strength properties of the casting [2]. We try not to let for these situations through the appropriate design of the casting mould enabling proper cooling of the casting and the suitable choice of the dimensions of the riser, what was taken up in this paper. One of the methods of evaluating the absence of shrinkage defects is continuous tracking of the position of the solidus line in the casting solidification process $[2,12]$. The smooth movement of this line (without separation) towards the riser where the process of solidification should end gives information about the casting made without shrinkage defects.

The destination of this paper was to choose the right dimensions and shape of the riser for solidifying the casting in a metal mould so that it would be formed without shrinkage defects. Assuming the conical or cylindrical riser shape, the numerical simulations were performed. The continuous moving of the solidus line was observed, in the following steps of the duration the process of directional solidification of the molten metal. If the end of the solidification occurred in the riser, it was assumed that the riser was chosen correctly and the casting remained without shrinkage defects.

\section{The mathematical and numerical model}

The proposed model for the numerical simulation of casting solidification is based on the solution of the Fourier-Kirchhoff equation [4-6, 10]:

$$
\nabla \cdot(\lambda \nabla T(\mathbf{x}, t))+\dot{Q}=\rho c \frac{\partial T}{\partial t},
$$

where: $\dot{Q}=\rho_{S} L \frac{d f_{S}(T(\mathbf{x}, t))}{d t}$ is the volumetric efficiency of the internal heat source $\left[\mathrm{W} / \mathrm{m}^{3}\right]$, $L$ - the latent heat of solidification $[\mathrm{J} / \mathrm{kg}], T$ - the temperature $[\mathrm{K}], f_{s}$ - volume fraction of solid phase $f_{s} \in[0,1], c$ - the specific heat $[\mathrm{J} /(\mathrm{kgK})], \lambda$ - the thermal conductivity coefficient $[\mathrm{W} /(\mathrm{mK})], \rho-$ the density $\left[\mathrm{kg} / \mathrm{m}^{3}\right], t-$ time $[\mathrm{s}], \mathbf{x}(x, y, z)-$ the coordinates of the vector of a considered node's position $[\mathrm{m}]$.

It was assumed that the solidification front is fuzzy, therefore the internal heat sources are not come evident in the equation of heat conductivity and differential equation (1) assumes in the form [4-6]:

$$
\nabla \cdot(\lambda \nabla T)-\rho C_{e f} \frac{\partial T}{\partial t}=0 .
$$

In the applied model of solid phase growth, the heat of phase transformation to the effective specific heat is introduced. Additionally, assuming the linear function of the solid phase fraction, the effective specific heat $\left(C_{e f}\right)$ for each phase is determined as follows $[4,6,10]$ : 


$$
C_{e f}(T)= \begin{cases}c_{L}(T), & T>T_{L}, \\ c_{L S}(T)+\frac{L}{T_{L}-T_{S}}, & T_{S}<T<T_{L}, \\ c_{S}(T), & T<T_{S} .\end{cases}
$$

where: $T_{L}, T_{S}$ is the temperature of liquids and solids line, respectively [K], $c_{L}, c_{L S}, c_{S}-$ the specific heat of liquid phase, mushy zone and solid phase, respectively $[\mathrm{J} /(\mathrm{kgK})]$.

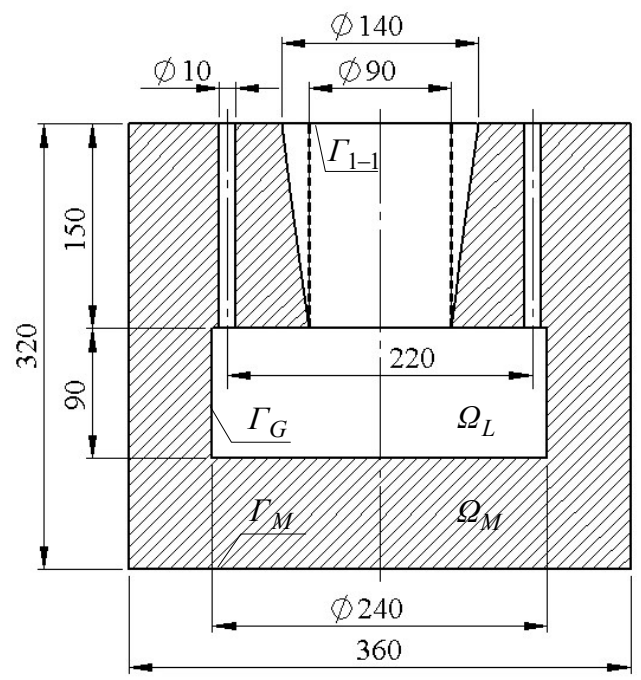

Fig. 1. The cross-section of the casting-mould system and identification of sub-regions of the considered region

The heat conduction equation (2) is completed by the appropriate initial conditions and the boundary conditions.

The initial conditions for temperature fields are given as [4-6]:

$$
T\left(\mathbf{x}, t_{0}\right)=T_{0}(x, y, z)=\left\{\begin{array}{lll}
T_{M} & \text { on } & \Gamma_{G} \\
T_{i n} & \text { in } & \Omega_{L} \\
T_{M} & \text { in } & \Omega_{M}
\end{array} .\right.
$$

The boundary conditions, on the indicated surfaces (Fig. 1), specified in the considered problem were as follows $[4,6,10]$ :

$$
\begin{array}{cc}
\left.\frac{\partial T}{\partial n}\right|_{\Gamma_{1-1}}=0, & \left.\lambda_{M} \frac{\partial T_{M}}{\partial n}\right|_{\Gamma_{M}}=-\alpha_{M}\left(\left.T_{M}\right|_{\Gamma_{M}}-T_{a}\right), \\
\left.\lambda_{S} \frac{\partial T_{S}}{\partial n}\right|_{\Gamma_{G-}}=\left.\lambda_{G} \frac{\partial T_{G}}{\partial n}\right|_{\Gamma_{G-}}, & \left.\lambda_{G} \frac{\partial T_{G}}{\partial n}\right|_{\Gamma_{G+}}=\left.\lambda_{M} \frac{\partial T_{M}}{\partial n}\right|_{\Gamma_{G+}},
\end{array}
$$

where: $T_{a}$ is the ambient temperature $[\mathrm{K}], T_{M}, T_{G}$ - the temperature of mould and gap (insulating coat), respectively $[\mathrm{K}], \lambda_{M}, \lambda_{G}, \lambda_{S}-$ the thermal conductivity coefficient of mould, gap and solid phase, respectively [K], $\alpha_{M}$ - the heat-transfer coefficient between mould and ambient $\left[\mathrm{W} /\left(\mathrm{m}^{2} \mathrm{~K}\right)\right]$. 
The numerical model uses finite element method in the weighted residuals formulation $[4-6,10]$. Equation (2) is multiplied by the weighting function $(N)$ and integrated over the entire region $(\Omega)$. As the result of using Ostrogradsky-Gauss-Green's theorem and Galerkin's method, which were described in the work [4], the final following matrix equation is obtained $[4-6,10]$ :

$$
\left(M_{K L}+K_{K L}\right) T_{L}^{s+1}=M_{K L} T_{L}^{s}+b_{K}^{s+1},
$$

where the elements of individual matrixes define integrals:

$$
\begin{aligned}
K_{K L} & =\int_{\Omega} \lambda N_{, j}^{K} N_{, j}^{L} d \Omega, \quad M_{K L}=\frac{1}{\Delta t} \int_{\Omega} \rho C_{e f} N^{K} N^{L} d \Omega, \\
b_{K} & =\int_{\Gamma} \lambda N^{K} T_{, j} n_{j} d \Gamma
\end{aligned}
$$

and these matrixes are called accordingly: $K_{K L}$ is the global thermal conductivity matrix, $M_{K L}$ - the global thermal capacity matrix, $b_{K}$ - global vector associated with the thermal boundary conditions, $T_{L}$ - vector of unknown nodal temperatures, $s$ - time level.

By solving of matrix equation (6) is obtained the sought field of temperature in the considered area.

\section{Examples of numerical calculations}

In this study, the analysis the effect of the shape change of riser on the temperature distribution in the three-dimensional system of casting-mould was made using the professional program SolidWorks Simulation. Transient analysis of the heat flow was carried out only by conduction but with the change of the cooled metal phase, modelled by temperature-dependent material properties in the liquid phase of the solidifying region. The calculations were performed for the longitudinal section shown in Figure 1 on a $2.4 \mathrm{GHz}$ IntelCore-i3 processor computer and lasted approximately 7 hours. The considered region has been divided into 545,299 tetrahedral finite elements that are defined by 742,553 mesh nodes. The mould is a cross section $360 \times 320$ and $360 \mathrm{~mm}$ in thickness and its internal surface is covered with an insulating coat. This insulating coat is made from a water suspension of quartzite dust with $2 \mathrm{~mm}$ thickness. The overall dimensions of the casting are equal to $\varphi 240 \times 90 \mathrm{~mm}$. Because, the computer calculations of the casting together with the conical or cylindrical riser are made, the dimensions of cylindrical riser amounts to $\varphi 90 \times 150 \mathrm{~mm}$ and conical riser are equal $\varphi 90 \times \varphi 140 \times 150 \mathrm{~mm}$.

The numerical calculations were made for low-carbon cast steel about chemical composition specified in work [1]. The thermophysical properties of cast steel were taken from works $[6,10]$. The changes in these parameters $(\rho, c, \lambda)$ with respect to temperature were taken into account. The overheated metal with temperature $T_{i n}=1850 \mathrm{~K}$ was poured into the steel mould with initial temperature $T_{M}=350 \mathrm{~K}$. The remaining characteristic temperatures were equal to: $T_{L}=1810 \mathrm{~K}, T_{S}=1760 \mathrm{~K}$ and $T_{a}=300 \mathrm{~K}$. The heat-transfer coefficient $(\alpha)$ between the mould and ambient was equal $\alpha_{M}=150 \mathrm{~W} /\left(\mathrm{m}^{2} \mathrm{~K}\right)$ and on the upper surface of the riser between the slag and ambient $\alpha_{s}=1 \mathrm{~W} /\left(\mathrm{m}^{2} \mathrm{~K}\right)$ [4-10].

Thermal phenomena, which proceeded in the mould cavity until total solidification of the casting (Figs. 2-12), were analysed. The influences of the shape change of riser on the temperature distribution in particular parts of the casting solidifying inside the steel mould on were determined. Examples of calculation results are shown in the form of temperature 
fields in Figures 2-9 for the casting together with the cylindrical riser or in Figures 10-12 for the conical riser. A continuous moving of the solidus line during the process of directional solidification of the molten metal was observed, because it gives information about forming the casting without shrinkage defects. If this line moves fluently to the riser, where the solidification process of the considered system should end, the casting free of defects will be created (Figs. 11, 12), if not, the defect will form in the casting (Figs. 8, 9).

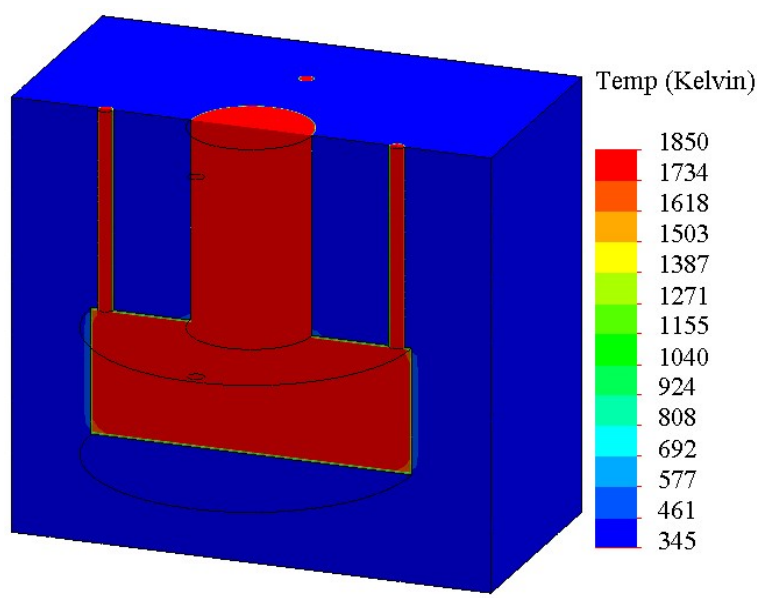

Fig. 2. Temperature distribution after time $10 \mathrm{sec}$

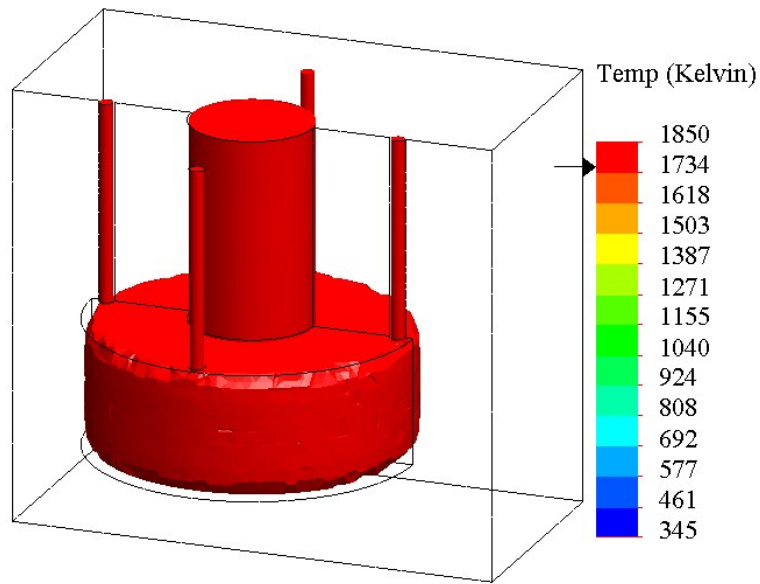

Fig. 3. Temperature field above the solidus temperature after $10 \mathrm{sec}$ 


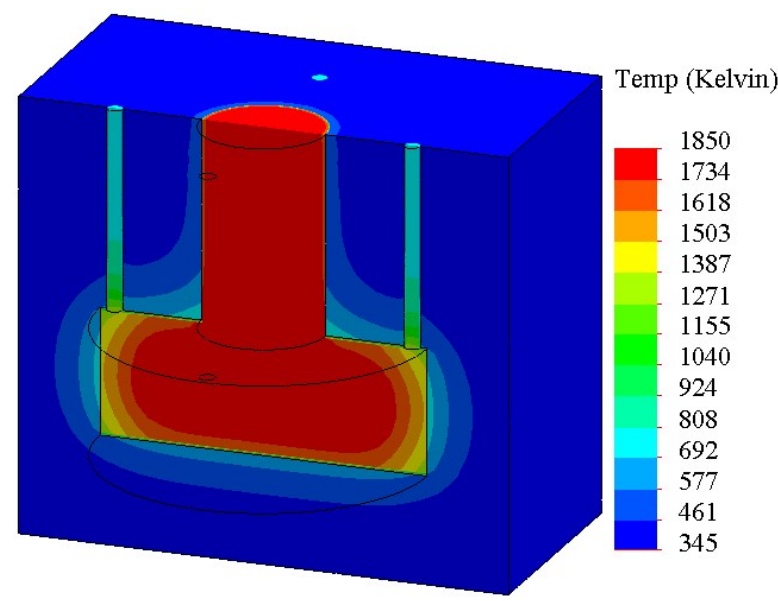

Fig. 4. Temperature distribution after time $100 \mathrm{sec}$

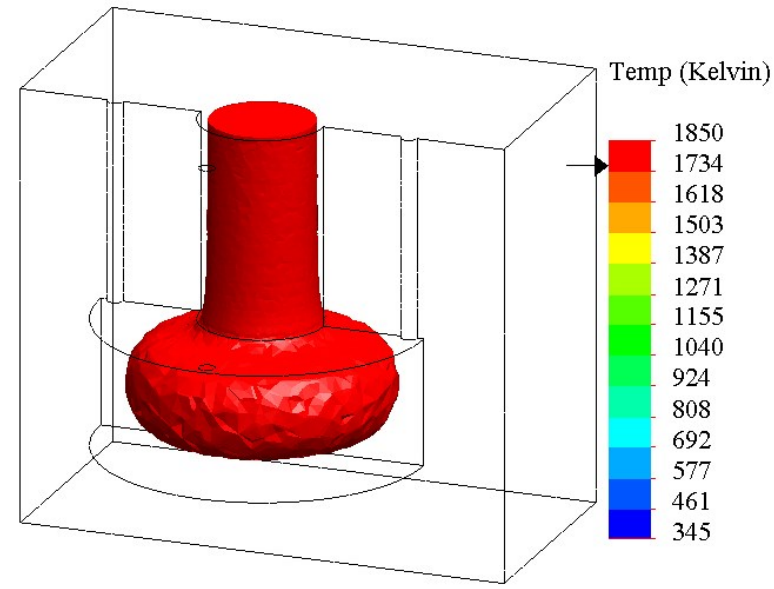

Fig. 5. Temperature field above the solidus temperature after $100 \mathrm{sec}$

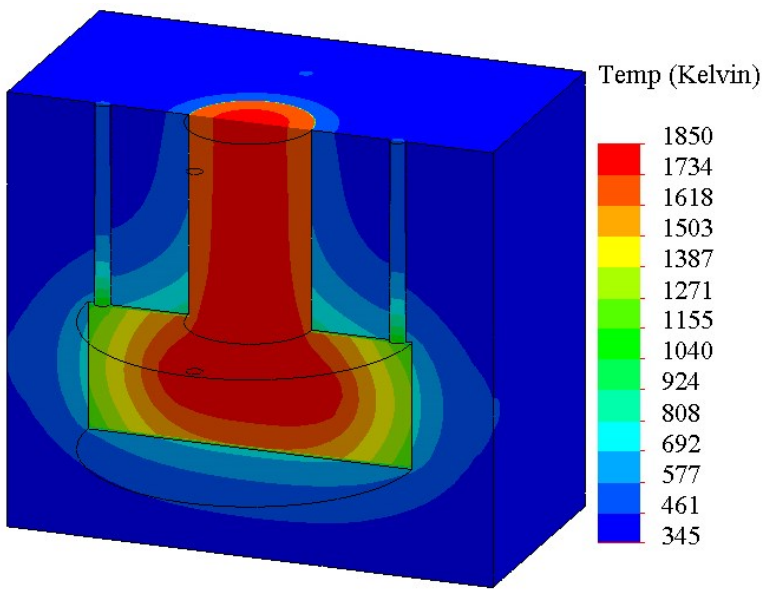

Fig. 6. Temperature distribution after time $200 \mathrm{sec}$ 


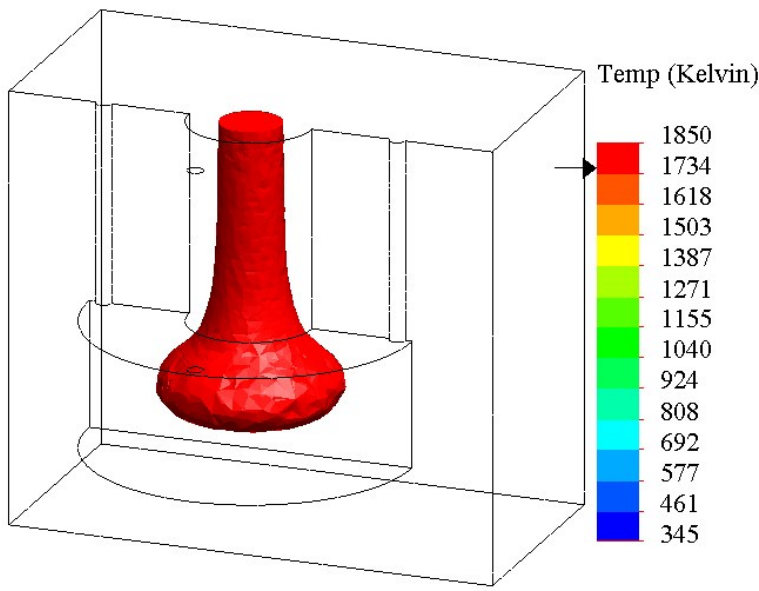

Fig. 7. Temperature field above the solidus temperature after $200 \mathrm{sec}$

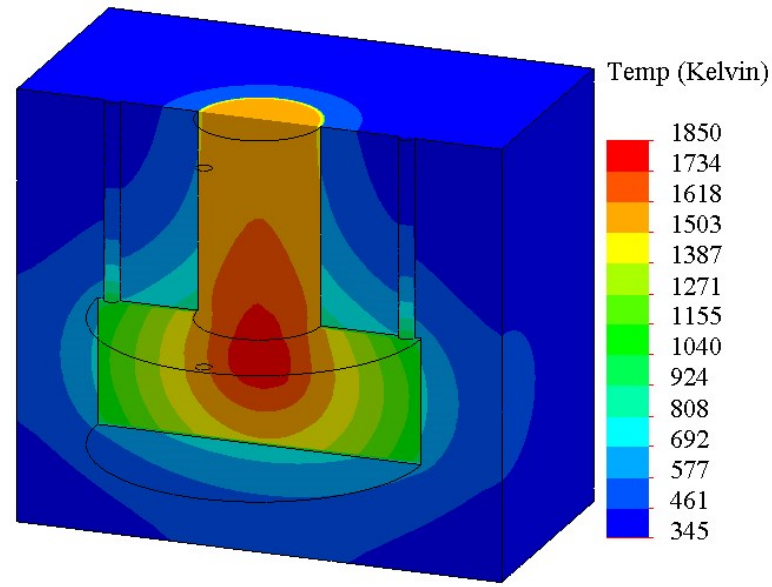

Fig. 8. Temperature distribution after time $330 \mathrm{sec}$

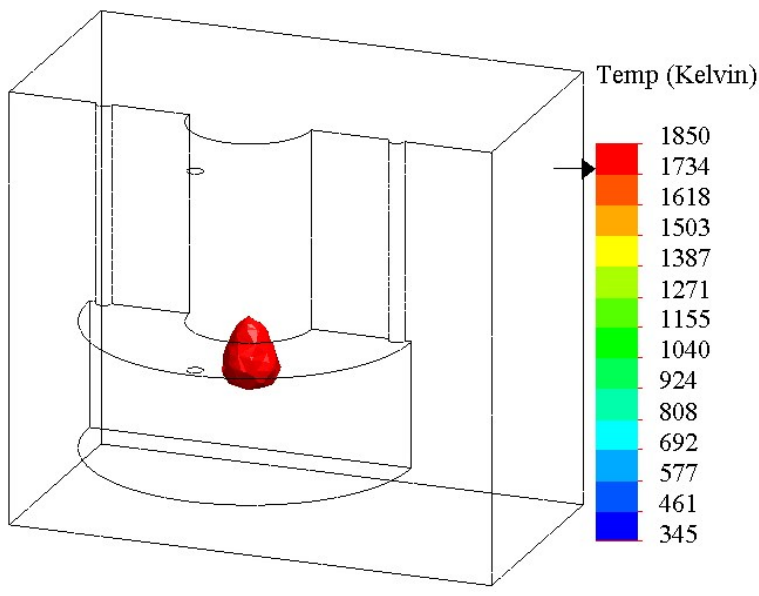

Fig. 9. Temperature field above the solidus temperature after $330 \mathrm{sec}$ 


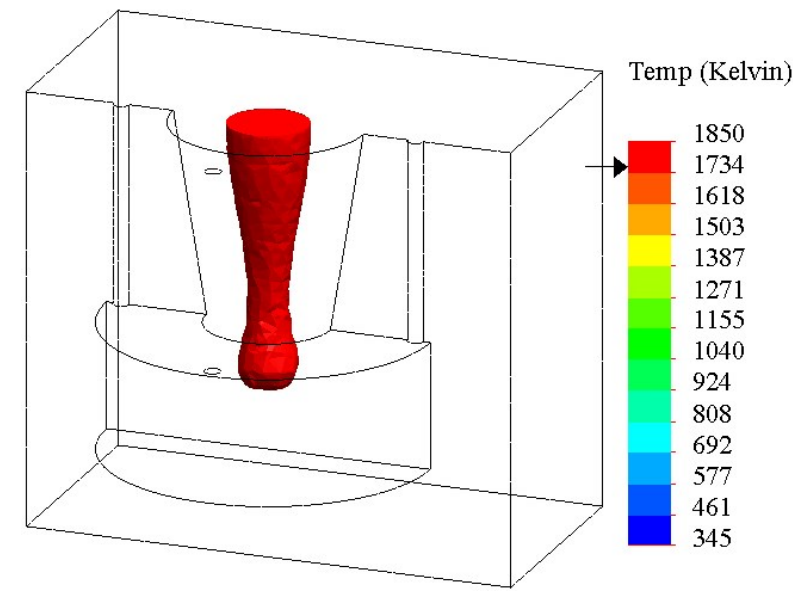

Fig. 10. Temperature field above the solidus temperature after $330 \mathrm{sec}$

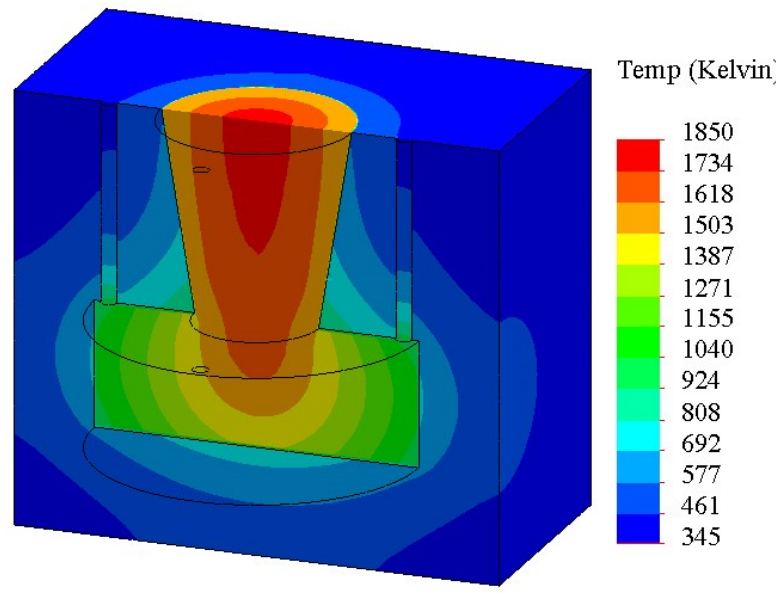

Fig. 11. Temperature distribution after time $380 \mathrm{sec}$

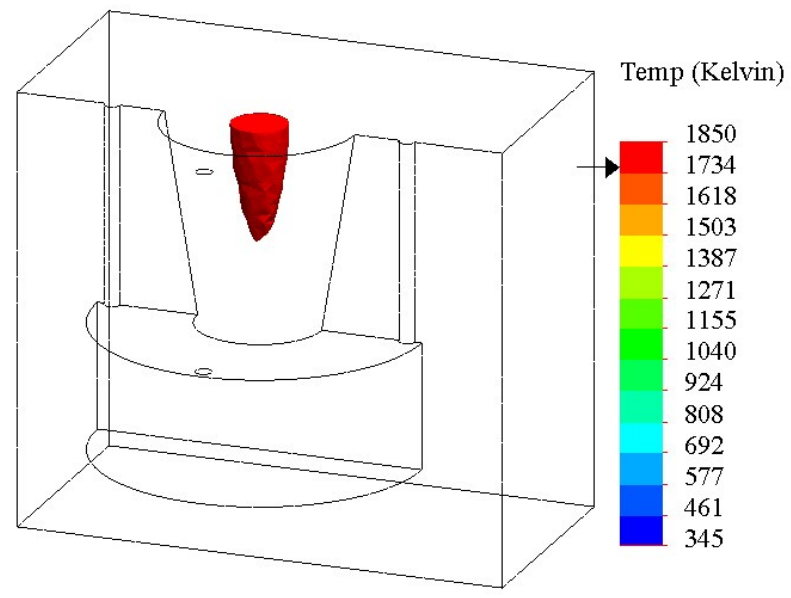

Fig. 12. Temperature field above the solidus temperature after $380 \mathrm{sec}$ 


\section{Conclusions}

The work concerns computer simulation of the unsteady process of the cast steel solidification in a metal mold using the finite element method. The solidification process of the molten metal in the three-dimensional cast-riser-mold system was analyzed, evaluating whether the conditions are conducive to the formation of shrinkage defects. Numerical calculations were made with the assumption of cylindrical or conical riser, obtaining temperature fields allowing follow the position of the solidus line in subsequent calculation stages (Figs. 2-12). It was observed whether this line is not closing disconnecting the solidifying casting into smaller areas with a difficult supply to them of liquid metal, because this would result in formation of shrinkage cavity in this place. This situation was not observed in the initial cooling period of the considered system, because there were favorable conditions for directional solidification (Figs. 2-7). In the final period solidification of the system casting-cylindrical riser, closing of the solidus line and location of the shrinkage cavity in the upper part of the casting was observed (Figs. 8, 9), which proves that such the riser did not fulfill its task. The change of the shape of riser to the conical riser, while maintaining the same dimension of its base, caused that the end of solidification occurred in the upper part of the riser, which is allowed, because the riser with the resulting shrinkage cavity is cut off and re-processed (Figs. 11, 12). Thus, the aim of this work was achieved because the right riser was chosen for the solidifying casting in a metal mold so that it was created without any shrinkage defects.

\section{References}

1. A. Walasek, J. Szajnar, The mechanism of the surface alloy layer creation for cast steel. Arch Foundry Eng. 12(1), 115-118 (2012)

2. Z. Ignaszak, J. Ciesiółka, Chosen identification problems of discontinuity in cast iron castings. Arch Foundry 4(14), 176-188 (2004)

3. M. Królikowski, A. Burbelko, D. Kwaśniewska-Królikowska, X-ray computed tomography in the nondestructive testing of ductile iron castings. Arch Foundry Eng. 14(4), 71-76 (2014)

4. T. Skrzypczak, E. Węgrzyn-Skrzypczak, L. Sowa, Numerical modeling of solidification process taking into account the effect of air gap. Appl Math Comput. 321, 768-779 (2018)

5. R. Dyja, N. Sczygiol, Method for determining the formation of shrinkage defects in the casting. Arch Foundry Eng. 11(4), 35-40 (2011)

6. T. Skrzypczak, E. Węgrzyn-Skrzypczak, Three-dimensional numerical model of solidification with motion of the liquid phase. Arch Foundry Eng. 11(2), 127-132 (2011)

7. M. Nadolski, A. Zyska, Z. Konopka, M. Łągiewka, J. Karolczyk, The assessment of bell casting producibility based on computer simulation of pouring and solidification. Arch Foundry Eng. 11(3), 141-144 (2011)

8. P-H. Huang, C-J. Lin, Computer-aided modeling and experimental verification of optimal gating system design for investment casting of precision rotor. Int J Adv Manuf Technol 79(7-8), 997-1006 (2015)

9. A. Gwiżdż, M Małysza, M. Nowak, Use of Flow-3D program for simulation of pouring and solidification process of ductile cast iron castings. Transactions of FRI 1, 3553(2013)

10. B. Mochnacki, S.J. Suchy, Numerical methods in computations of foundry processes. (Polish Foundrymen's Technical Association, Kraków, 1995 
11. T. Skrzypczak, E. Węgrzyn-Skrzypczak, L. Sowa, Computer Simulation of the Solidification Process Including Air Gap Formation. Arch Foundry Eng 17(4), 147-150 (2017)

12. C. M. Choudhari, B. E. Narkhede, S. K. Mahajan, Casting design and simulation of cover plate using AutoCAST-X software for defect minimization with experimental validation. Proc Mat Scien. 6, 786-797 (2014) 